\title{
Eficiencia hospitalaria en regiones colombianas con percepción de corrupción, mediante técnica DEA
}

\section{Hospital efficiency in Colombian regions where corruption is evident by means of DEA technique}

INFORMACIÓN DEL

\section{ARTÍCULO}

Fecha de recepción: 5 de Abril de 2021. Fecha de aceptación: 9 de Junio de 2021.

${ }^{1}$ Estudiante de la Maestría en Ciencias Económicas y de Gestión, Pontificia Universidad Javeriana Cali.

E-mail: yeariasg@javerianacali.edu.co Código ORCID:

https://orcid.org/0000-0003-1995-5184

${ }^{2}$ Magíster en Ciencias Económicas y de Gestión, Pontificia Universidad Javeriana Cali. Investigador, Pontificia Universidad Javeriana Cali - Colombia.

E-mail: agustinru@javerianacali.edu.co Código ORCID:

https://orcid.org/0000-0002-0220-554X

CITACIÓN: Arias Galindo, YE, \& Ramírez Urraya, A. (2021). Eficiencia hospitalaria en regiones colombianas con percepción de corrupción, mediante técnica DEA. Podium, 39, 83-102.

doi:10.31095/podium.2021.39.6

\section{ENLACE DOI:}

http://dx.doi.org/10.31095/podium.202 1.39 .6

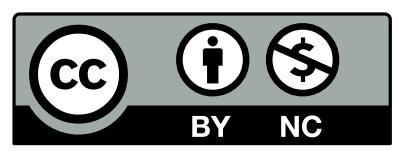

Yeick Exneider Arias Galindo ${ }^{1}$, Agustín Ramírez Urraya ${ }^{2}$

\section{Resumen}

La eficiencia es un tema importante a la hora de analizar el sector público, y de mayor interés cuando se mira hacia el sector salud. El objetivo de este estudio es identificar la eficiencia de los hospitales públicos de nivel I en los departamentos de Córdoba, Sucre y Valle del Cauca, dada su alta percepción de corrupción y, con ello, ubicarlos en cuartiles. Se obtuvo una base de datos con 612 registros de hospitales públicos de nivel I de los 32 departamentos de la República de Colombia, de los cuales se filtraron los mismos bajo criterios de percepción de corrupción. Se realizó un estudio empírico mediante análisis envolvente de datos -DEA-. Como resultado, los hospitales más eficientes pertenecen al departamento de Córdoba y los más ineficientes son los del departamento del Valle del Cauca.

\section{Palabras Clave:}

Colombia, eficiencia hospitalaria, análisis envolvente de datos, sector salud, hospital público, percepción de corrupción.

Clasificación JEL: H21, H51, C14.

\begin{abstract}
Efficiency is an important issue when analyzing the public sector, and of greater interest in the health sector. This study purports to identify level I public hospitals' efficiency in the Córdoba, Sucre and Valle del Cauca departments, given their high corruption perception and thereby locate them in quartiles. A database was obtained with 612 records from level I public hospitals in the 32 Republic of Colombia departments; they were filtered under corruption perception criteria. An empirical study was carried out using data envelopment analysis -DEA-. As a result, the most efficient hospitals are in the Córdoba department and the least efficient are in the Valle del Cauca department.
\end{abstract}

\section{Keywords:}

Colombia, hospital efficiency, data enveloping analysis, health sector, public hospital, corruption perception.

JEL Classification: H21, H51, C14. 


\section{Introducción}

En las últimas décadas, el sector de la salud en Colombia ha confrontado algunos cambios de gran importancia - como la reforma a la salud, supeditada al carácter institucional y al ejercicio de la no transparencia en sus procesos administrativos y financieros-, que como consecuencia han tenido grandes efectos y repercusiones sobre la prestación de servicios, la variación de las finanzas del sistema de salud tendientes a ser negativa, y la estructura financiera de los hospitales públicos subsanados por el Estado ante su insolvencia (Suárez-Rozo, Puerto-García, Rodríguez-Moreno, y Ramírez-Moreno, 2017).

En los hospitales y el sistema de salud en general, estudios sobre producción, y en particular los que abarcan la eficiencia y productividad, exponen retos de especial horizonte para cualquier interesado. Retos constituidos en la complejidad de los sistemas de producción y la diferencia dada a partir de los productos e insumos para el sector salud con respecto de otros, cuestiones éstas que atribuyen restricciones para el análisis de la temática (Nupia y Sánchez, 2001). La eficiencia y la productividad de este tipo de organizaciones son aspectos de sumo valor para ser analizados, persiguiéndose con ello el hecho de que dicho sector económico pueda lograr competitividad.

Así, surge la necesidad de analizar la eficiencia de los hospitales públicos teniendo presente el uso de sus insumos y la oferta de servicios que estos brindan a la ciudadanía, representando ello la unidad estructural de costos e ingresos, pudiendo resultar de dicho análisis aportes de interés para la construcción e implementación de estrategias para el sector salud.

El presente trabajo se asienta en marcos teórico-conceptuales de la denominada eficiencia como enfoque de medida relativa, que busca describir la relación dada entre los insumos con los productos, y ello con respecto de los valores máximos posibles del proceso de obtención de las determinadas condiciones tecnológicas (Biltrán y Valor-Sabatier, 1987; Farrell, Grosskopf, Lindgren, y Roos, 1995) y, por otro lado, el DEA como un enfoque que basado en la programación lineal, busca medir la eficiencia relativa de las Unidades de Toma de Decisión -DMU- escogidas a analizar (Charnes, Cooper, y Rhodes, 1978). El análisis de eficiencia hospitalaria se ha desarrollado en algunos estudios desde la década de los 80 mediante la técnica conocida como Data Envelopment Analysis -DEA- (Hollingsworth, 2008).

Entrando en materia, la intención de este estudio es analizar las eficiencias de los hospitales públicos de nivel I de los departamentos de Cordoba, Sucre y Valle del Cauca (Colombia), dada su condición de alta percepción de corrupción, ubicándoles en cuartiles representativos. Y, a partir de ello, comprender los apartados que apoyen la toma de decisiones con el objeto de lograr mayor eficiencia en el sector económico de la salud.

El artículo se compone de cinco 
apartados, la introducción siendo la primera. Posteriormente se aborda una breve revisión de literatura, abordando la relación entre la eficiencia hospitalaria y la corrupción en el sector salud. En el tercer apartado se expone la metodología, donde se presenta la técnica utilizada, las variables seleccionadas -con su data-, y el modelo matemático. El cuarto apartado, refleja los resultados descriptivos. Por último, se dan a conocer unas breves conclusiones, limitaciones y líneas nacientes de investigación.

\section{Revisión de literatura}

Eficiencia hospitalaria y corrupción en la salud: Una breve reseña de su relación

En el mundo la salud es un servicio esencial y de vital importancia, instituido para la contribución de la mejoría en la calidad de vida de las personas (Castro y Tapia, 2021; San-José, Retolaza y Bernal, 2021), siendo así también en Colombia. Dado ello, es necesario generar estudios que giren sobre la estructura económica abarcando las variables relacionadas con sus insumos y productos, permitiéndose analizar la situación de los mismos y que, como respuesta, fundamente decisiones que favorezcan la optimización de los recursos de estas entidades (Navarro, Maza y Viana, 2012).

El análisis de eficiencia es una de las herramientas más usadas por diseñadores de políticas en los sistemas de salud (Santelices et al., 2013). Los gastos en salud como proporción relativa de la producción van en incremento, originando nuevos desafíos para los países en vía de desarrollo (Castro y Tapia, 2021). Es de tener en cuenta que una importante parte de dichos recursos públicos para la salud son consumidos por hospitales, y como resultado, un gran número de investigadores generan interés en su análisis, concentrando esfuerzos de estudio a ese nivel (Lee, Langdo, Hwang, Marques, y Hwang, 2021).

Ejemplificando, un hospital convendría tener capacidad de solventar problemas específicos del ámbito de la salud -aun requiriendo tecnologías y personal especializado-, y contar con recurso sin importar que los requerimientos se den en escasos casos, dicha noción de ser contraria podría vérsele como una ineficiencia (Puig-Junoy, 2000). Así, la eficiencia es entonces relativa entre los distintos hospitales, por lo cual para este estudio se asumirá que un hospital tiende a ser menos eficiente si éste necesita grandes cantidades de insumos para producir la misma cantidad de bienes y servicios que otro hospital que ejecuta menos recursos (Mora y Morales, 1997).

Para el caso colombiano, la eficiencia demostrada en hospitales es bastante criticada. Llinás (2010) halló que el modelo colombiano es observa $\neg$ do como poco satisfactorio frente a otros países, $\mathrm{y}$ advierte que la eficiencia del sistema de salud falla cuando ésta ha emanado en administraciones improductivas, en demoras en los flujos financieros y en el uso clandestino de los recursos, que han dado lugar a resultados desalentadores del carácter que arraigan resultados 
nefastos. Según Navarro y Hernández (2002) el éxito y superación de las políticas públicas del sector salud dirigidas hacia mejores prácticas, deben pasar en primera medida por la iden-tificación de forma oportuna de todos aquellos servicios que son eficientes diferenciándolos de los ineficientes.

Por otro lado, la literatura abordada en temáticas sobre la no transparencia en los procesos y su incidencia en la economía de la salud demuestra que la corrupción conlleva a una alta reducción de la eficiencia institucional de muchas de estas entidades, afectando así a importantes variables de su medición -como la inversión- y propiamente al crecimiento y desarrollo económico tanto de las regiones como de las entidades mismas (Tanzi y Davoodi, 1997; Kaufmann, 1998; Bigio y Ramírez, 2006; Akcay, 2006).

Los gobiernos municipales de Colombia inspeccionan y conceden subsidios, además de contribuir con la mejoría de la cobertura de la salud, enfocados a las ciudadanías de escasos recursos. Dicho dispositivo de estipendio y concesión de recursos públicos aumenta los intereses de los privados, captando el interés público de la búsqueda por el aumento de provisión en salud. Esto afecta la eficiencia sin importar la cantidad de hospitales y, por ende, las conexiones en red de proveedores de salud (Giménez, Prieto, Prior y Tortosa-Ausina, 2019).

Giménez et al. (2019) expresan que las políticas públicas orientadas a proteger y fomentar la cobertura, pueden estar aumentando la tentación de captura de las instituciones gubernamentales locales de la salud por parte de personas privadas, impulsando con ello el mal uso de los gastos de los hospitales públicos, debiéndose esto a la corrupción observada en la gestión de los recursos públicos para expresos niveles de resultados administrativos y financieros.

En el contexto internacional, han sido demostrados los vastos efectos inscritos como malignos donde la corrupción incidió, inyectándose en las esferas estructurales de la política, la economía y lo social (Sommersguter-Reichmann y Stepan, 2015). Ante ello, Ortiz (2012) afirma que Colombia es uno de los países latinoamericanos ejemplo de una de las más altas aceptaciones acerca de los efectos perjudiciales y desfavorables de la corrupción, además de la necesidad de enfrentarla.

La noción de la corrupción ha sido un problema persistente en la historia con amplios efectos nocivos, señalándosele como uno de las principales limitantes de la economía y del bajo desarrollo de las regiones. Estudios exponen la dimensión de las causas y consecuencias del fenómeno, donde Colombia se potencializa con problemáticas varias, que van desde narcotráfico, ineficiencia del sistema estatal, ilegalidad y delitos públicos, indebidos procesos, y otras más (Ortiz, 2012).

Lo anterior pone de manifiesto la relevancia de este estudio dado el carácter de estigma de la percepción de 
corrupción en regiones colombianas. El desarrollo de la presente investigación es procedido teniendo como base datos de los hospitales públicos del nivel I de las municipalidades de tres departamentos que, al periodo 2016-2018, son catalogados como los de más alta percepción de corrupción en el país. Se aclara que la noción de la corrupción no es tomada como variable en sí, pero apoya la fundamentación de la selección de las regiones de donde se analizan los datos.

\section{Metodología}

Aspectos metodológicos para la medición de la eficiencia hospitalaria

La eficiencia según García (2002) tiene bastantes perspectivas que tienden a ser diferentes entre sí, identificándose el considerar distintas alternativas y posibilidades para su cálculo dentro de un grupo de entidades. Ligarda y Naccha (2006) exponen que el uso eficiente de los recursos es determinante, y más cuando se trata de servicios de salud que tienen incidencia en el bienestar de los individuos. Como complemento, Chilingerian (1995) define la eficiencia como toda aquella capacidad de producir bienes y servicios con el mínimo nivel de recursos posible.

En sumatoria, la técnica DEA permite analizar niveles de eficiencia $\mathrm{y}$, desde modelaciones, tomar decisiones que busquen optimizar el uso de recursos. Pinillos (2003) plantea que el DEA es importante cuando los niveles de productividad dependen de diferentes compromisos, tales como el personal administrativo y operativo, el liderazgo, las decisiones, entre otros; y más aún cuando se trata de la prestación de servicios en entidades de salud.

Es significativo entonces precisar que los niveles de eficiencia obedecerán a las variables de medición seleccionadas, es decir, dependen de las entradas y salidas a evaluarse. Otra noción de lo anterior es la propuesta por Gedion y Morales (1999) donde estipulan que la eficiencia de una entidad de salud depende de dos aspectos principales: del uso que se brinde a sus insumos $\mathrm{y}$ de su costo. El primero frecuentemente es conocido como eficiencia técnica (García, 2002), relaciona los insumos utilizados en razón de los productos y servicios que se generan.

Por otro lado, al evaluarse la eficiencia se debe considerar también los costos de los factores asociados con las condiciones de presta $\neg$ ción del bien, así como los escenarios requeridos para la dismi $\neg$ nución de sus costos. Esta idea ha sido abarcada por autores en sus estudios, entendiéndola como eficiencia económica o eficiencia asignativa (Sánchez, Nupia y Urdinola, 2000; García, 2002). Para la aplicación de lo anterior se cuenta con métodos sofisticados, como la estimación de fronteras y de la eficiencia, que además difieren de otros métodos que solo miden y comparan comúnmente los comportamientos observados.

Una entidad del sector servicios para ser eficiente debe, además de potenciar el rendimiento de su capital humano y tecnológico, propiciar el aumento de los 
niveles de su productividad tanto como de su eficacia, relacionadas éstas con los insumos o entradas para la prestación del servicio, de tal manera que dicha entidad pueda competir y ajustarse a las condiciones impuestas por las nuevas circunstancias de la economía (Martínez et al., 2002).

A pesar de lo anterior, un hecho que genera atención es el que se da cuando en dos estudios, aunque tienen originalmente el mismo ámbito de acción, sus resultados tienen diferencias respecto de las causas de la ineficiencia. Cuestión esta que permite orientar la enunciación de que las causas de un uso no óptimo de los recursos pueden estar dadas por los factores externos a la entidad, así como también por los mismos entes (influencia política, corrupción, procesos indebidos, etc.) hasta por las decisiones internas que son ineficientes. Pese a lo anterior, otro hecho que suma atención aquí es el de la calidad, que no tiene porqué chocar con la búsqueda de la reducción de costos. Este aspecto de calidad es raramente evaluado en los diferentes enfoques de estudios de la economía de la salud, efecto debido a notorios limites frente a la obtención de datos para dicha variable.

El DEA en la medición de la eficiencia hospitalaria

Los modelos DEA han sido una de las técnicas más recurrentes, especialmente para la medición de la eficiencia en el sector salud por una gran variedad de autores, entre los que cabe mencionar a Banker, Conrad y Strauss (1986),
Grosskopf y Valdmanis (1987), Byrnes y Valdmanis (1995), Chilingerian (1995), Farell et al. (1995), Sodani y Madnani (2008).

El DEA permite construir, con base en la información real de los insumos utilizados por cada hospital y sus productos, los niveles de la eficiencia para cada una de las entidades estudiadas. El resultado de estos ejercicios permite identificar a los hospitales eficientes e ineficientes; estos últimos siendo aquellos que, dada su utilización de insumos otorgados, se hallan ubicados por debajo del nivel de producción que potencialmente podrían estar ejecutando.

Es de resaltar que al estudiarse temas de analisis de eficiencia pueden ser consideradas dos opciones. La primera es la orientación hacía insumos (input-orientation), la cual expone como supuesto el hecho de que una empresa conservando un nivel de producción constante, debería responderse a sí misma cuál es el nivel óptimo de insumos por contratar para ser eficiente. Y la segunda opción es la orientación hacía el producto (output-orientation), cuestión que pretende dar respuesta a cuál es el nivel de producción óptimo que hace que una empresa sea eficiente frente a otras defendiendo su nivel actual de consumo de insumos.

También es importante destacar la existencia de dos supuestos respecto a los retornos que ostentan las unidades productivas. El primero de que las empresas operan a escala óptima y que, por ende, atribuye la objetividad de 
rendimientos constantes a escala (RCE). $\mathrm{Y}$, en contraparte, el segundo supone que las fallas de mercado -competencia imperfecta, restricciones financieras, etc- causan que no todas las empresas operen a óptima escala, y frente a ello, los rendimientos a escala varían entre empresas, conocido esto como rendimientos variables a escala (RVE).

Se plantea entonces el supuesto de que cada uno de los $n$ hospitales de los departamentos de Cordoba, Sucre y Valle del Cauca utilizan una cierta cantidad de $K$ insumos determinados para producir una cantidad dada de $M$ productos explícitos para un período de tiempo específico. Lo que alude al hecho de que en el i-ésimo hospital, una cierta cantidad de insumos contratados y de unidades producidas son representadas por los vectores $\mathrm{x}_{\mathrm{i}} \quad\left(K x_{1}\right) \quad$ y yi $\left(M x_{1}\right)$, respectivamente. De dicha forma, la matriz Kxn de insumos $(X)$, y la matriz Mxn de productos $(Y)$, representan todos los datos dados para el total de los hospitales.

Así, aplicándose un enfoque de orientación de insumos y de rendimientos constantes a escala (RCE), se busca hallar para cada hospital la combinación de insumos que maximice la relación producto por factor. Lo anterior se expresaría como un problema de optimización de la siguiente forma (Modelo 1):

$$
\begin{aligned}
\operatorname{Max}_{\mu, v} & \left(\mu^{\prime} y_{i}\right)^{\prime} \\
\text { S.a. } & v^{\prime} x_{i}=1 \\
& \mu^{\prime} y_{\mathrm{j}}-v^{\prime} x_{j} \leq 0, j=1,2, \ldots, N \\
& \mu, v \geq 0
\end{aligned}
$$

Donde $\mu$ es el vector $\left(M x_{1}\right)$ de combinación óptima de productos, y el vector $\left(K x_{1}\right)$ que representa la combinación óptima de factores a ser halladas sería v. Con esto se busca hallar, los valores dados para $\mu$ y $v$, tales que orienten la medida de la eficiencia del $i$-ésimo hospital hacia el ser maximizada, sujeta a que esta medida deba ser menor o igual a uno (1). Dicha implicación está supeditada a la primera y la segunda restricción, por lo que el ejercicio se puede proyectar como $\operatorname{Max}_{\mu, v}\left(\mu^{\prime} y_{i}, v^{\prime} x_{i}\right)$ sujeto a que $\mu^{\prime} y_{i} / v^{\prime} x_{i} \leq 1$ y $\mu, v \geq 0$. No obstante, para evitar casos de obtener soluciones infinitas se atribuye que $v^{\prime} x_{i}=1$.

De igual forma, la eficiencia puede también ser subdividida en dos importantes bloques: la eficiencia técnica y la eficiencia asignativa. La primera representa todas las habilidades que tiene una empresa para lograr un nivel máximo de producción radicado a sus combinaciones de insumos. El segundo refleja la habilidad dada por una empresa para lograr la utilización recomendable de insumos como proporción óptima dados sus precios referidos. Se debe tener en cuenta que, para medir esta última eficiencia, el modelo debe introducir los precios de los factores. El planteamiento de dicho modelo, con RVE, puede plantearse de la siguiente forma (Modelo 2):

$$
\begin{aligned}
\operatorname{Min}_{\lambda \mathrm{xi}^{*}} & w_{\mathrm{i}}{ }^{\prime} x_{\mathrm{i}}^{*} \\
\text { S.a. } & -y_{\mathrm{i}}+Y \lambda \geq 0 \\
& x_{\mathrm{i}}^{*}-X \lambda \geq 0 \\
& N_{1}^{\prime} \lambda=1 \\
& \lambda \geq 0
\end{aligned}
$$

Donde wi representa al vector que 
determina el precio de los insumos para el i-ésimo hospital y xi* es el vector que determina los insumos que minimizan los costos ceñido a los precios de los factores integrados y el nivel de producción yi. Estas medidas pueden reflejarse de una forma más clara en la Figura 1.

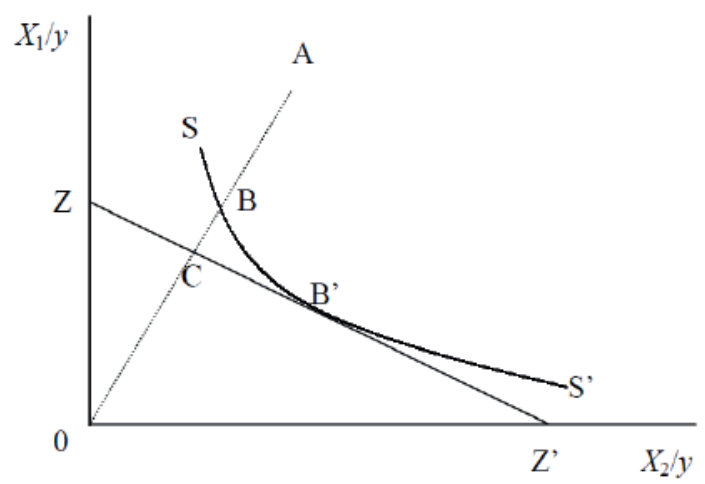

Figura 1. Modelo de maximización de insumos por costos. Fuente: Nupia y Sánchez, 2001.

Observando la Figura 1, suponga que se tiene una empresa que utiliza una combinación de insumos dados, definida por el punto $\mathrm{A}$, que proceden para la producción de una unidad de producto. Dicha empresa podría producir la misma cantidad de producto con algunas combinaciones de menos factores. El punto $\mathrm{B}$ es el punto de eficiencia técnica para esta empresa -vista sobre la curva isocuanta $\mathrm{SS}^{\prime}-$, por lo que su ineficiencia técnica se halla dada por la diferencia entre la distancia existente en los puntos A y B. Pese a ello, en el punto observado $\mathrm{B}$, se podría deducir que la empresa está produciendo con una combinación de factores de cáracter asignativa inadecuado. Lo anterior se debe a que en el punto $\mathrm{B}$, la productividad marginal del factor $\mathrm{X}_{2}$ representa una disminución frente a su costo marginal (observar la isocosto constituida por la línea que une los puntos ZZ'). Es posible diferenciarse las dos medidas adicionales. La primera diferencia está dada por la ineficiencia puramente asignativa que se observa en la distancia entre los puntos $\mathrm{B}$ y $\mathrm{C}$, representando así una reducción en los costos debiendo ocurrir ésta para que la empresa llegue al punto de producción $\mathrm{B}^{\prime}$. Y, por último, se tiene la ineficiencia económica total $-\mathrm{O}$ reducción de costos-, que permite incluir tanto a la ineficiencia técnica como la asignativa, esta ineficiencia se puede medir por la diferencia dada en la distancia entre los puntos A y C.

\section{Material de trabajo: Data y variables}

El tercer informe de la Corporación Transparencia por Colombia (2019) afirma que cerca del $56 \%$ del total de los hechos de corrupción analizados entre 2016-2018, afectaron el sector de educación (16\%), infraestructura y transporte (15\%), de la Salud (13\%) y de la Función Pública (12\%). Esta situación coincide con los sectores que mayormente son afectados y que se exponen en el informe sobre los hechos proyectados en Corrupción en Territorios de Paz (2010-2016). Lo anterior permite ostentar la idea de que donde están los recursos económicos, allí estarán los intereses de los corruptos.

Ahora bien, cifras de los últimos años de los presupuestos generales de la nación permiten evidenciar que los sectores salud y educación son los que obtienen una mayor inversión de los recursos estatales. En salud, por ejemplo, el presupuesto en promedio destinado es del $25 \%$ del presupuesto general de la 
nación (Corporación Transparencia por Colombia, 2019). Con todo ello, da pie para hacerse a imaginarios de asignación no transparentes que además han sido reflejados en algunas estadísticas reportadas.

Asimismo, frente a la percepción de corrupción para el sector salud son los departamentos de Sucre (14\%), Córdoba (14\%) y Valle del Cauca (11\%) donde se reportan la mayoría de los hechos. Es importante hacer mención a casos como el cartel del sida (2018), el de los enfermos mentales (2012), el de la hemofilia (2013) y hasta el cartel de las cirugías (2013). Los anteriores casos son los vistos como los más criticos de la salud ocurridos en Sucre y Córdoba (Corporación Transparencia por Colombia, 2019). Cuestión ésta que brinda soporte para querer realizar estudio enfocado en estos tres departamentos colombianos.

Las premisas antes mencionadas permiten ubicar el contexto respecto de la selección de los departamentos a los que pertenecen las entidades hospitalarias públicas de nivel I (también conocidas como DMUs en los estudios de eficiencia) a ser analizadas. Las estimaciones se realizan utilizando la información de dichos hospitales públicos nivel I para el año 2013, los datos son provenientes del Sistema de Información Hospitalaria (SIHO) del Ministerio de Salud y de la Protección Social de Colombia.

\section{Variables de ingreso (Inputs)}

Maldonado y Tamayo (2007) explican que si bien para incorporar las variables de trabajo laboral se hace uso de forma frecuente el número de personal, representado en los médicos, las enfermeras y el personal administrativo, en ocasiones es dificultoso realizar segregación de ellos debido a la deficiencia de información correspondiente al personal externo contratado (servicios indirectos) donde solo se reporta el valor total de dichas contrataciones, debiéndose tener en cuenta que el gasto en servicios indirectos supera -en promedio- a los gastos del personal de planta. Ante dicha limitación, se trabaja entonces con los gastos de nómina.

Así, las variables seleccionadas como insumos/inputs son aquellas que se suelen utilizar para demostrar la producción de servicios en salud. Se entiende por insumos en salud a aquellos donde se cuenta con la intervención de personal necesario para brindar la atención pertinente y oportuna al paciente que demanda el servicio, además de los utensilios necesarios para suplir dicha demanda, incluyendo los medicamentos.

Por lo cual, son cuatro (4) las variables aquí: compras de bienes y servicios (que incluye el material mobiliario y de oficina, material para uso documental, software, entre otros), compras de medicamentos, gastos de personal administrativo y los gastos de personal operativo -en esta última categoría ingresan los profesionales de la medicina y personal de enfermería-. Diferentes autores han usado estas variables para medir la eficiencia hospitalaria (Banker et al., 1986; Biltrán 
y Valor-Sabatier, 1987; Prior y Solà, 2000; Solà y Prior, 2001; Biorn, Hagen, Iversen, y Magnussen., 2003; Pinzón, 2003; Peñaloza, 2003; Fontalvo y Dela Hoz, 2016).

\section{Variables de salida (Outputs)}

Las entidades hospitalarias nivel I -también denominados como de baja complejidad-, suelen ser el primer contacto de los ciudadanos con los servicios de salud. Estas entidades requieren de baja tecnología, simple de usar, para la atención de problemas de la salud de escasa gravedad, pero que son de impacto en los resultados tanto como en los costos. En estas entidades se cuenta con un factor humano de tipo profesional y técnico general. Además, se atienden principalmente a casos ambulatorios y hospitalizaciones no graves (Pinzón, 2003).

Pinzón (2003) expone que, frecuentemente se ha denotado que el principal objetivo de toda organización con fin de prestación de servicios de salud es proporcionar productos como egresos de resultado preciso. En esos casos, los egresos son entendidos como la cantidad de tratamientos ofrecidos a pacientes que han ingresado al hospital con alguna patología. La Tabla 1 expone cada una de las variables seleccionadas para el presente estudio, además presenta una breve definición para las mismas.

Así, la variable utilizada como producto es la representada como el objeto esencial de los servicios de las instituciones de baja complejidad. Para todas las instituciones de la muestra se tiene el número de casos tratados ponderados por complejidad que es expresado como la producción. Pinzón (2003) indica que los casos tratados como producto de un hospital están asociados a los servicios hospitalarios, mediante el cual los pacientes permanecen por un mínimo periodo de tiempo mientras es tratado por el personal de la salud (diagnóstico, terapia, cirugía ambulatoria en casos, medicamentos, etc.) y luego se retira. Esta variable es la mayoritariamente usada por varios autores para el análisis de eficiencia (Borden, 1990; Ozcan, Luke, y Haksever,

Tabla 1.

Función de producción de hospitales nivel I - 2013 (Descripción de variables seleccionadas)

\begin{tabular}{|c|c|c|}
\hline Tipo & Variables & Definición \\
\hline $\begin{array}{c}\text { Producto } \\
\text { (Output) }\end{array}$ & Producción & Casos tratados ponderados por \\
\hline & Compras_ByS & complejidad del caso (No casos) \\
\hline Insumos & Medicamentos & Compras de bienes y servicios (COP) \\
\hline (Inputs) & Gasto_Admón & Compras de medicamentos (COP) \\
& Gastos_Asistencial & Gasto personal administrativo (COP) \\
& & Gasto personal asistencia - médicos y \\
\hline
\end{tabular}

Fuente: Elaboración propia. 
1992; Prior y Solà, 2000; Solà y Prior, 2001; Biorn et al., 2003; Wang, Zhao, y Mahmood, 2006).

\section{Resultados}

Aquí se evidencian las estadísticas descriptivas de las variables analizadas para proceder con un breve acercamiento sobre los hospitales públicos de nivel I pertenecientes a los municipios de los departamentos con mayor porcentaje de percepción de corrupción en Colombia. En general, los presentes resultados permiten ubicar a los hospitales de dichos municipios categorizándolos en cuartiles según es su eficiencia hospitalaria dada por sus inputs y outputs (ver Anexos 1 y 2).

A partir del modelo DEA, se logró obtener los cálculos de las eficiencias para los hospitales de nivel I en los tres (3) departamentos con mayor porcentaje de percepción de corrupción en el país, y posteriormente se logra reseñar las unidades más eficientes que son referencia con respecto de las no eficientes. En las estadísticas se demuestra que existe una vasta dispersión en la magnitud de los datos, denotando esto que las medidas obtenidas para el coeficiente de variación tienen una tendencia de máximos y una gran diferencia entre el grupo mismo, donde se puede observar la distancia entre la medida obtenida para la variable Compras_ByS y Producción determinando que, previo a los análisis matemáticos sofisticados, existe una mala gestión de los recursos.

Por otro lado, se observa en la Tabla 2 que los rubros de mayor relevancia para los hospitales de nivel I son los relacionados con las compras de bienes y servicios y las de medicamentos, con el $51.97 \%$ y el $45.19 \%$ respectivamente; en tanto, el rubro de importancia menor es el gasto asistencial con el $1.37 \%$. En promedio, para los hospitales pertenecientes a los departamentos estudiados, se registra un valor de inversión por caso tratado de $6143.84 \mathrm{COP}$.

Se agruparon los municipios de hospitales públicos nivel I por cuartiles según criterio de medición de la eficiencia siendo Q1 los hospitales ubicados en los municipios donde se observa la existencia de mayor nivel de eficiencia. Se resaltan los primeros cinco (5) hospitales más eficientes que, a saber,

Tabla 2.

Estadísticas descriptivas de variables consideradas en el estudio

\begin{tabular}{|c|c|c|c|c|}
\hline Variables & Media & Mediana & Desv. Estándar & Coef. Varianza \\
\hline Compras_ByS & 783982133.7 & 448351078.8 & 1047769463.4 & 1097820848483620000.0 \\
\hline Medicamentos & 681810187.2 & 303416775.7 & 1036584676.2 & 1074507790990930000.0 \\
\hline Gto_Admón & 22193047.0 & 15348789.0 & 26957237.6 & 726692660622622.0 \\
\hline Gto_Asistencial & 20674129.7 & 17643530.0 & 13879698.4 & 192646027958303.0 \\
\hline Producción & 245556.3 & 157467.2 & 262649.9 & 68984945693.6 \\
\hline
\end{tabular}

Fuente: Elaboración propia. 
pertenecen a los municipios de Montería, Planeta Rica, Pueblo Nuevo, Tierralta y Valencia, es de recalcar de nuevo que los municipios estudiados pertenecen a los tres departamentos con mayor percepción de corrupción según la Corporación Transparencia por Colombia (2019). Mientras tanto, los cinco (5) municipios con hospitales de resultados de menor eficiencia son Buenaventura, Dagua, San Marcos, Sucre y La Unión.

La tabla 3 evidencia la agrupación dada a los hospitales de nivel I ubicados en los municipios de los departamentos con más alta percepción de corrupción. Para ello, el Anexo 1 permite realizar la asignación en cuartiles, donde la columna Eficiencia apoya el ejercicio de asignar los mismos. Entendido, todos aquellos valores entre $0,751-1,00$ se asumen como hospitales con alta eficiencia, asimismo, se entiende por hospitales con eficiencia moderadamente alta a aquellos con valores entre $0,51-0,750$. De igual manera, valores entre $0,251-0,50$ son tenidos en cuenta como hospitales con eficiencia moderadamente baja, y los hospitales con eficiencia baja son los que se ubican entre los valores $0,00-0,250$.

Por último, es importante mencionar que las demás columnas en el Anexo 1 reflejan los valores en término de exponer cuánto son eficientes cada hospital de nivel I con cada uno de sus inputs, y cuánto son eficientes en su output a partir de la combinación de todos sus inputs. También se presenta el Anexo 2 que brinda la información utilizada -base de datos discriminada por variable - para cada uno de los inputs y el output de los hospitales nivel I de los municipios de departamentos con la más alta percepción de corrupción del país.

\section{Conclusiones}

Cumpliendo con el objetivo del presente estudio, el cual es identificar la eficiencia de los hospitales públicos de nivel I de los municipios de los

Tabla 3.

Distribución por cuartiles según eficiencia de los hospitales nivel I -municipios-.

\begin{tabular}{|c|c|}
\hline Cuartil & Municipios \\
\hline $\begin{array}{c}\text { Q1 } \\
(0,751-1,00)\end{array}$ & $\begin{array}{r}\text { Montería, Planeta Rica, Pueblo Nuevo, Tierralta, Valencia, Sincelejo, Jaimito, } \\
\text { Colorado, El Roble, San Benito de Abad, San Juan de Vetulia, San Pedro (S.), } \\
\text { Cali, Alcalá, Argelia, El Cerrito, Jamundí, San Pedro (V.), Puerto Escondido, } \\
\text { Vijes, Toro, Buenavista, Tuluá, Palmito, Galeras, Restrepo, Cartago, Yumbo. }\end{array}$ \\
\hline $\begin{array}{r}\text { Q2 } \\
(0,51-0,750)\end{array}$ & $\begin{array}{r}\text { La Victoria, El Cairo, Ciénaga de Oro, San Luis de Sincé, Yotocó, La } \\
\text { de Buga, Andalucía, Canalete, Florida, Trujillo, Ginebra, Palmira, Sampués, } \\
\text { San Onofre, Montelíbano, Caicedonia, Ansermanuevo, Tolú Viejo, } \\
\text { Candelaria, El Dovio, Majagual. }\end{array}$ \\
\hline $\mathbf{Q 3}$ & $\begin{array}{r}\text { Calima, La Unión, Sucre, San Marcos, Dagua, Buenaventura. } \\
(0,251-0,50)\end{array}$ \\
\hline $\mathbf{Q 4}$ & N. A. \\
\hline
\end{tabular}

Fuente: Elaboración propia. 
departamentos de Córdoba, Sucre y Valle del Cauca, dada su alta percepción de corrupción, y ubicarlos en cuartiles (de Q1 a Q4), se concluye entonces a partir de los resultados obtenidos que los hospitales del departamento de Córdoba son los más eficientes de entre los tres departamentos estudiados, para su administración durante el año 2013, aun cuando se ha dicho que éste es uno de los departamentos pertenecientes a los elegidos por el criterio de mayor percepción de corrupción en el país.

Igualmente, es de resaltarse que las tres ciudades capitales de dichos departamentos arrojaron resultados positivamente satisfactorios frente a sus valores de eficiencia. Los hospitales de nivel I pertenecientes al departamento de Sucre obtuvieron resultados moderados en sus niveles de eficiencia. La mayoría de los hospitales correspondientes al departamento del Valle del Cauca son los que podría catalogárseles como los de menores niveles de eficiencia. Es de recordar que para la presente investigación por eficiencia se entiende que una empresa, conservando sus niveles de producción constante, usa sus insumos al nivel más óptimo posible buscando hallar la combinación de insumos que maximice la relación producto por factor.

Por otro lado, la mayor limitación presentada en este estudio fue la falta de algunas variables importantes en la base de datos obtenida -percepción de corrupción por municipio, percepción de calidad en producto y servicio, entre otras de interés-, cuestión que amarra la investigación a los datos mismos. Se recomienda entonces como horizontes de investigación realizar estudios que abarquen análisis transversales donde éste permita observar la relación del crecimiento de la eficiencia a través del tiempo, además expandir las investigaciones a otras esferas geográficas donde se tenga también en cuenta el criterio de selección territorial con respecto de la percepción de la corrupción, realizando comparaciones.

Para finalizar, una interesante línea de más sería realizar estudios longitudinales donde se puedan obtener resultados con datos más actualizados para las mismas variables, e incorporar otras que permitan obtener mayor rigurosidad en la profundidad de los análisis. Además, es importante realizar estudios donde se incorporen variables de calidad para dichos territorios catalogados como de mayores procesos de corrupción en la salud frente a territorios con baja percepción de procesos de corrupción, $\mathrm{y}$ hasta quizá extender dichos análisis al ámbito de lo internacional. También sería bien estudiar la integración de diferentes variables de entorno que permitan obtener datos respecto de la corrupción como una variable moderadora del proceso de eficiencia en un hospital. Sumado, realizar análisis para hospitales de diferentes niveles de complejidad en su oferta de servicios.

\section{Referencias}

Akcay, S. (2006). Corruption and Human Development. Cato Journal, 26(1). Cato Institute, Turkey.

Banker, R., Conrad, R., y Strauss, R. (1986). A comparative application of data 
Yeick Exneider Arias Galindo, Agustín Ramírez Urraya

envelopment analysis and translog methods: an illustrative study of hospital production. Management Science, 32(1), $30-44$.

Bigio, S., y Ramírez, N. (2006). Corrupción e Indicadores de Desarrollo: Una Revisión Empírica. Banco Central de Reserva del Perú. Lima, Perú.

Biltrán, G., y Valor-Sabatier, J. (1987). Some mathematical programming based measures of efficiency in health care institutions. Advances in Mathematical Programming and Financial Planning, (1), 61-84.

Biorn, E., Hagen, T., Iversen, T. y Magnussen, J. (2003). The effect of activity-based financing on hospital efficiency: A panel data analysis of DEA efficiency scores 1992-2000. Health Care Management Science, 6(4), 271-283.

Borden, J. (1990). An assessment of the impact of diagnosis-related group (DGR)-based reimbursement on the technical efficiency of New Jersey hospitals using data envelopment analysis. Journal of Accounting and Public Policy, (7), 77-96.

Byrnes, P., y Valdmanis, V. (1995). Analyzing technical and allocative efficiency of hospital. In Charnes, A., Cooper, W., Lewin, W., Arie Y., Seiford, Lawrence, M. (Ed.). Data Envelopment Analysis: Theory, Methodology and Applications. Boston, EU.

Castro, R. y Tapia, J. (2021). Adding a social risk adjustment into the estimation of efficiency: the case of Chilean hospitals. Quality Management in Health Care, 30(2), 104-111.

Charnes, A., Cooper, W. y Rhodes, E. (1978). Measuring the efficiency of decision making units. European Journal of Operational Research, 2(6), 429-444.
Chilingerian, J. (1995). Exploring why some physicians' hospital practices are more efficient: taking DEA inside the hospital. In Charnes, A.,

Cooper, W., Lewin, W., Arie Y., Seiford, Lawrence, M. (Ed.). Data Envelopment Analysis: Theory, Methodology and Applications. Boston, EU.

Corporación Transparencia por Colombia (2019). Así se mueve la corrupción: Radiografía de los hechos de corrupción en Colombia 2016-2018. Monitor Ciudadano de Corrupción, Tercer Informe.

Farrell, R., Grosskopf, S., Lindgren, B., y Roos, P. (1995). Productivity developments in Swedish Hospital: A malmquist output index approach. In Charnes, A., Cooper, W., Lewin, W., Arie Y., Seiford, Lawrence, M. (Ed.). Data Evelopment Analysis: Theory, Methodology and Applications. Boston, EU.

Fontalvo, T., y Dela Hoz (2016). Eficiencia de los hospitales de Bolívar, Colombia, por medio análisis envolvente de datos. Dimensión Empresarial, 14(1), 95-108.

García, C. (2002). Análisis de la eficiencia técnica y asignati $\neg$ va a través de las fronteras estocásticas de costes: una aplica $\neg$ ción a los Hospitales del INSALUD. Tesis Doctoral. Universidad de Valladolid. Valladolid: España.

Gedion, U., y Morales L. (1999). Aproximación a la medición de la eficiencia económica y eficiencia de la gestión en los Hos $\neg$ pitales públicos del Distrito Capital. Fedesarrollo y Secretaría Distrital de Salud. Bogotá: Colombia.

Giménez, V., Prieto, W., Prior, D. y Tortosa-Ausina, E. (2019). Evaluation of efficiency in Colombian hospitals: An analysis for the post-reform period. Socio-Economic Planning Sciences, (65), 20-35. 
Grosskopf, S., y Valdmanis, V. (1987). Measuring hospital performance: a non-parametric approach. Journal of Health Economics, (6).

Hollingsworth, B. (2008). The measurement of efficiency and productivity of health care delivery. Health Economics, 17(10), 1107-1128.

Kaufmann, D. (1998). Corrupción y reforma institucional: el poder de la evidencia empírica. Presentado en la 1a Conferencia Perspectivas, Instituto del Banco Mundial. Washington, EU.

Lee, Cs., Langdo, J., Hwang, D., Marques, V., y Hwang, P. (2021). Impacts of distributors and group purchasing organizations on hospital efficiency and profitability: a bilateral data envelopment analysis model. International Transactions in Operational Research, 28(1).

Ligarda, J., y Ñaccha, M. (2006). La eficiencia de las organiza $\neg$ ciones de salud a través del análisis envolvente de datos. Micro-redes de la Dirección de Salud IV Lima Este 2003. An Fac Med Lima, 67(2), 142-151.

Llinás, A. (2010). Evaluación de la calidad de la atención en salud: Un primer paso para la reforma del sistema de la salud. Revista Cientifica Salud Uninorte, 26(1), 143-154.

Maldonado, A., y Tamayo, N. (2007). Estudio integral de eficiencia de los hospitales públicos. Archivos de Economía, DNP. Documento 338.

Martínez, S., Rubiera, F., Cueto, B., Dávila M., Quindós, M., y Vicente, M. (2002). Los servicios avanzados a empresas en el Principado de Asturias: situación actual y perspectivas futuras. Instituto de Desarrollo Económico del Principado de Asturias. Asturias: España.

Mora, H., y Morales, L. (1997). Consideraciones sobre la evaluación de la eficiencia relativa de los hospitales colombianos.
Universitas Económica, 9, 21-39.

Navarro, J. y Hernández, E. (2002). ¿Es posible relacionar la calidad y la eficiencia de los Hospitales públicos? En Universidad de Granada (Ed.). Granada: España.

Navarro, J., Maza, F., y Viana, R. (2012). La eficiencia de los hospitales colombianos en el contexto latinoamericano. Una aplicación de Análisis Envolvente de Datos (DEA) en un grupo de hospitales de alta complejidad, 2009. Ecos de Economía, 15(33), 71-99.

Nupia, O., y Sánchez, F. (2001). Eficiencia de los hospitales públicos de Bogotá. Desarrollo y Sociedad, (48), 101-136.

Ortiz, E. (2012). Efectos de la corrupción sobre la calidad de la salud y la educación en Colombia 2004-2010. TENDENCIAS Revista de la Facultad de Ciencias Económicas y Administrativas, 13(1), 9-35.

Ozcan, Y., Luke, R. y Haksever, C. (1992). Ownership and organizational performance. A comparison of technical efficiency across hospital types. Medical Care, (30), 781-94.

Pinillos, M. (2003). Eficiencia del servicio público de atención sanitaria primaria. Papeles de Economía Española, (95), 213-225.

Peñaloza, M. (2003). Evaluación de la Eficiencia en instituciones hospitalarias públicas y privadas con Data Envelopment Analysis (DEA). Archivos de Economía, DNP. Documento 244.

Pinzón, M. (2003). Medición de eficiencia técnica relativa en hospitales públicos de baja complejidad mediante la metodología Data Envelopment Analysis (DEA). Archivos de Economía, DNP. Documento 245.

Prior, D., y Solà, M (2000). Technical efficiency and economies of diversification in health care. Health Care Management Science, 
(3), 299-307.

Puig-Junoy, J. (2000). Efficiency in primary health care: a critical survey of the frontier approach. Revista Española de Salud Pública, 74(5-6), 483-495.

Sánchez, F., Nupia, O., y Urdinola, P. (2000). Gasto público: Efi $\neg$ ciencia y cobertura del sector salud. Calidad de la inversión en ca $\neg$ pital humano. Misión de Reforma Institucional de Bogotá, Secretaría de Hacienda. Bogotá, Colombia.

San-José, L., Retolaza, J., y Bernal, R. (2021). Social value added index: a proposal for analyzing hospital efficiency. Gaceta Sanitaria, 35(1), 21-27.

Santelices, E., Ormeño, H., Delgado, M., Lui, Ch., Valdés, R., y Durán, L. (2013). Análisis de la eficiencia técnica hospitalaria 2011. Revista Médica de Chile, (141), 332-337.

Sodani, P., y Madnani, G. (2008). Measuring Hospital Performance through Data Envelopment Analysis: Understanding Basic Concepts to Help Novice Researchers. Journal of Health Management, 10(1), 129-142.

Solà, M., y Prior, D. (2001). Measuring productivity and quality changes using data envelopment analysis: an application to catalan hospitals. Financial Accountability \& Management, 17(3), 219-45.

Sommersguter-Reichmann, M., y Stepan, A. (2015). The interplay between regulation and efficiency: Evidence from the Austrian hospital inpatient sector. Socio-economic Planning Sciences, (52), 10-21.

Suárez-Rozo, L., Puerto-García, S., RodríguezMoreno, L., y Ramírez-Moreno, J. (2017). La crisis del sistema de salud colombiano: Una aproximación desde la legitimidad y la regulación. Rev Gerenc Polit Salud, 16(32), 34-50.
Tanzi, V., y Davoodi, H. (1997). Corruption, public investment and growth. IMF Working Paper, WP/97/139. Paper presentado en el 53o Congreso del Instituto Internacional de Finanza Pública Kyoto, Japón.

Wang, J., Zhao, Z. y Mahmood, A. (2006). Relative Efficiency, Scale Effect, and Scope Effect of Public Hospitals: Evidence from Australia. IZA, Discussion Paper, $\mathrm{N}^{\mathrm{o}}$ 2520, Bonn, Alemania. 
Eficiencia hospitalaria en regiones colombianas con percepción de corrupción, mediante técnica DEA

\section{Anexos}

Anexo 1.

Eficiencia y medidas de eficiencia de cada variable para el total de las DMUs

\begin{tabular}{|c|c|c|c|c|c|c|c|}
\hline Municipios & DMU_list & Eficiencia & Compras_ByS & Medicamentos & Gto_Admón & Gto_Asistencial & Producción \\
\hline MONTERÍA & Branch_1 & 1 & 0 & $8.35 \mathrm{E}-11$ & $2.57 \mathrm{E}-08$ & 0 & $8.69 \mathrm{E}-07$ \\
\hline CANALETE & Branch_2 & 0.643201579 & $9.26 \mathrm{E}-10$ & 0 & 0 & 7.13E-08 & $2.76 \mathrm{E}-06$ \\
\hline CIÉNAGA DE ORO & Branch_3 & 0.726066119 & $5.03 \mathrm{E}-10$ & 0 & $2.69 \mathrm{E}-08$ & $2.34 \mathrm{E}-08$ & $1.72 \mathrm{E}-06$ \\
\hline MONTELÍBANO & Branch_4 & 0.57673627 & $1.33 \mathrm{E}-10$ & $7.43 \mathrm{E}-11$ & $3.11 \mathrm{E}-08$ & $9.47 \mathrm{E}-09$ & $1.21 \mathrm{E}-06$ \\
\hline PLANETA RICA & Branch_5 & 1 & $9.07 \mathrm{E}-10$ & $2.03 \mathrm{E}-11$ & 0 & $1.36 \mathrm{E}-08$ & $2.04 \mathrm{E}-06$ \\
\hline PUEBLO NUEVO & Branch_6 & 1 & $2.29 \mathrm{E}-09$ & $5.76 \mathrm{E}-11$ & $3.61 \mathrm{E}-08$ & 0 & $5.11 \mathrm{E}-06$ \\
\hline PUERTO ESCONDIDO & Branch_7 & 0.986811631 & 0 & $1.82 \mathrm{E}-10$ & $1.91 \mathrm{E}-07$ & 0 & 0 \\
\hline TIERRALTA & Branch_8 & 1 & $2.68 \mathrm{E}-10$ & $9.51 \mathrm{E}-14$ & 0 & 7.54E-09 & 7.26E-07 \\
\hline VALENCIA & Branch_9 & 1 & $2.88 \mathrm{E}-10$ & $1.80 \mathrm{E}-10$ & $5.66 \mathrm{E}-08$ & 0 & $2.50 \mathrm{E}-06$ \\
\hline SINCELEJO & Branch_10 & 1 & $1.03 \mathrm{E}-10$ & $1.82 \mathrm{E}-10$ & $1.23 \mathrm{E}-08$ & 0 & $1.66 \mathrm{E}-06$ \\
\hline BUENAVISTA & Branch_11 & 0.870912004 & $1.28 \mathrm{E}-09$ & $2.77 \mathrm{E}-11$ & $6.26 \mathrm{E}-08$ & $1.00 \mathrm{E}-08$ & 0 \\
\hline CAIMITO & Branch_12 & 1 & $1.14 \mathrm{E}-09$ & 4.69E-09 & 0 & $2.51 \mathrm{E}-08$ & $3.44 \mathrm{E}-06$ \\
\hline COROZAL & Branch_13 & 1 & $6.98 \mathrm{E}-10$ & $5.69 \mathrm{E}-11$ & 0 & $3.48 \mathrm{E}-08$ & 2.27E-06 \\
\hline EL ROBLE & Branch_14 & 1 & $2.49 \mathrm{E}-09$ & $2.27 \mathrm{E}-09$ & 4.69E- 08 & 0 & 0 \\
\hline GALERAS & Branch_15 & 0.82295476 & $1.84 \mathrm{E}-09$ & 5.33E-09 & 0 & 0 & $3.89 \mathrm{E}-06$ \\
\hline MAJAGUAL & Branch_16 & 0.510530685 & 0 & $3.20 \mathrm{E}-10$ & $6.82 \mathrm{E}-08$ & 0 & $1.75 \mathrm{E}-06$ \\
\hline MORROA & Branch_17 & 1 & $1.92 \mathrm{E}-09$ & $1.59 \mathrm{E}-11$ & 7.82E-08 & 0 & 0 \\
\hline PALMITO & Branch_18 & 0.829550022 & 2.04E-09 & $1.99 \mathrm{E}-10$ & 4.54E- 08 & $1.31 \mathrm{E}-09$ & $2.91 \mathrm{E}-06$ \\
\hline SAMPUÉS & Branch_19 & 0.599002472 & $4.71 \mathrm{E}-10$ & $6.91 \mathrm{E}-10$ & $2.91 \mathrm{E}-08$ & 0 & $1.64 \mathrm{E}-06$ \\
\hline SAN BENITO ABAD & Branch_20 & 1 & $1.56 \mathrm{E}-09$ & $3.51 \mathrm{E}-11$ & 0 & $2.35 \mathrm{E}-08$ & $3.52 \mathrm{E}-06$ \\
\hline $\begin{array}{c}\text { SAN JUAN DE } \\
\text { BETULIA }\end{array}$ & Branch_21 & 1 & $1.46 \mathrm{E}-09$ & $3.18 \mathrm{E}-11$ & 7.19E-08 & $1.15 \mathrm{E}-08$ & 0 \\
\hline SAN MARCOS & Branch_22 & 0.416184136 & $3.76 \mathrm{E}-10$ & $5.52 \mathrm{E}-10$ & $2.33 \mathrm{E}-08$ & 0 & $1.31 \mathrm{E}-06$ \\
\hline SAN ONOFRE & Branch_23 & 0.592701533 & 0 & $3.29 \mathrm{E}-10$ & 7.01E-08 & 0 & $1.80 \mathrm{E}-06$ \\
\hline SAN PEDRO & Branch_24 & 1 & $1.99 \mathrm{E}-09$ & $1.30 \mathrm{E}-10$ & $2.77 \mathrm{E}-08$ & 2.61E-09 & 4.64E-06 \\
\hline SAN LUIS DE SINCÉ & Branch_25 & 0.724324663 & $6.09 \mathrm{E}-10$ & $4.75 \mathrm{E}-09$ & $5.94 \mathrm{E}-09$ & 0 & 2.39E-06 \\
\hline SUCRE & Branch_26 & 0.421342854 & 0 & $5.31 \mathrm{E}-09$ & $5.80 \mathrm{E}-09$ & 0 & $1.28 \mathrm{E}-06$ \\
\hline SANTIAGO DE TOLÚ & Branch_27 & 0.653222294 & $7.00 \mathrm{E}-10$ & $1.03 \mathrm{E}-09$ & 4.33E-08 & 0 & 2.44E-06 \\
\hline TOLÚ VIEJO & Branch_28 & 0.539213212 & $2.13 \mathrm{E}-09$ & $1.29 \mathrm{E}-09$ & $1.28 \mathrm{E}-08$ & 0 & $3.60 \mathrm{E}-06$ \\
\hline CALI & Branch_29 & 1 & $6.68 \mathrm{E}-10$ & $1.72 \mathrm{E}-10$ & 0 & $7.59 \mathrm{E}-10$ & $1.54 \mathrm{E}-06$ \\
\hline ALCALÁ & Branch_30 & 1 & $2.22 \mathrm{E}-09$ & $3.38 \mathrm{E}-11$ & 2.64E-08 & $1.65 \mathrm{E}-08$ & $3.89 \mathrm{E}-06$ \\
\hline ANDALUCÍA & Branch_31 & 0.646744973 & $1.66 \mathrm{E}-09$ & $1.07 \mathrm{E}-09$ & $8.74 \mathrm{E}-09$ & 0 & $2.88 \mathrm{E}-06$ \\
\hline ANSERMANUEVO & Branch_32 & 0.556884382 & $7.75 \mathrm{E}-10$ & $1.08 \mathrm{E}-10$ & $2.50 \mathrm{E}-08$ & $1.09 \mathrm{E}-08$ & $1.34 \mathrm{E}-06$ \\
\hline ARGELIA & Branch_33 & 1 & $1.92 \mathrm{E}-09$ & $1.59 \mathrm{E}-11$ & 7.82E-08 & 0 & 0 \\
\hline BOLÍVAR & Branch_34 & 0.694728569 & $1.10 \mathrm{E}-09$ & 0 & 0 & $8.46 \mathrm{E}-08$ & $3.28 \mathrm{E}-06$ \\
\hline BUENAVENTURA & Branch_35 & 0.361874801 & $4.74 \mathrm{E}-10$ & $6.00 \mathrm{E}-11$ & 3.23E-08 & 0 & 0 \\
\hline $\begin{array}{c}\text { GUADALAJARA DE } \\
\text { BUGA } \\
\end{array}$ & Branch_36 & 0.652712186 & $5.23 \mathrm{E}-10$ & $6.98 \mathrm{E}-11$ & 0 & 2.36E-08 & $1.57 \mathrm{E}-06$ \\
\hline CAICEDONIA & Branch_37 & 0.572696052 & $9.45 \mathrm{E}-10$ & $8.64 \mathrm{E}-11$ & $2.98 \mathrm{E}-09$ & $1.02 \mathrm{E}-08$ & $2.00 \mathrm{E}-06$ \\
\hline CALIMA & Branch_38 & 0.495363889 & $1.27 \mathrm{E}-09$ & $1.19 \mathrm{E}-10$ & $2.82 \mathrm{E}-08$ & 0 & $1.75 \mathrm{E}-06$ \\
\hline CANDELARIA & Branch_39 & 0.537099507 & 2.17E-10 & $1.96 \mathrm{E}-10$ & $3.55 \mathrm{E}-08$ & 0 & $1.28 \mathrm{E}-06$ \\
\hline CARTAGO & Branch_40 & 0.814576135 & $9.03 \mathrm{E}-10$ & 0 & 0 & $1.50 \mathrm{E}-08$ & $2.11 \mathrm{E}-06$ \\
\hline DAGUA & Branch_41 & 0.412190435 & $5.65 \mathrm{E}-10$ & $7.55 \mathrm{E}-11$ & 0 & $2.55 \mathrm{E}-08$ & $1.70 \mathrm{E}-06$ \\
\hline EL ÁGUILA & Branch_42 & 0.688541718 & $1.01 \mathrm{E}-09$ & $2.19 \mathrm{E}-11$ & 4.95E-08 & 7.94E-09 & 0 \\
\hline
\end{tabular}


Yeick Exneider Arias Galindo, Agustín Ramírez Urraya

\begin{tabular}{|c|c|c|c|c|c|c|c|}
\hline Municipios & DMU_list & Eficiencia & Compras_ByS & Medicamentos & Gto_Admón & Gto_Asistencial & Producción \\
\hline EL CAIRO & Branch_43 & 0.743761759 & $1.14 \mathrm{E}-09$ & $3.15 \mathrm{E}-09$ & 0 & $2.77 \mathrm{E}-08$ & 0 \\
\hline EL CERRITO & Branch_44 & 1 & $1.43 \mathrm{E}-09$ & $1.26 \mathrm{E}-10$ & $6.66 \mathrm{E}-10$ & $1.48 \mathrm{E}-08$ & $2.96 \mathrm{E}-06$ \\
\hline EL DOVIO & Branch_45 & 0.532382532 & $1.31 \mathrm{E}-09$ & $1.28 \mathrm{E}-10$ & $2.93 \mathrm{E}-08$ & $8.44 \mathrm{E}-10$ & $1.88 \mathrm{E}-06$ \\
\hline FLORIDA & Branch_46 & 0.631511266 & $4.12 \mathrm{E}-10$ & $1.18 \mathrm{E}-10$ & $2.69 \mathrm{E}-08$ & $1.10 \mathrm{E}-08$ & $1.61 \mathrm{E}-06$ \\
\hline GINEBRA & Branch_47 & 0.617850222 & $1.36 \mathrm{E}-09$ & $1.33 \mathrm{E}-10$ & $3.04 \mathrm{E}-08$ & $8.76 \mathrm{E}-10$ & $1.95 \mathrm{E}-06$ \\
\hline GUACARÍ & Branch_48 & 0.702581198 & $1.36 \mathrm{E}-09$ & $7.91 \mathrm{E}-10$ & $1.35 \mathrm{E}-08$ & $4.94 \mathrm{E}-09$ & $2.76 \mathrm{E}-06$ \\
\hline JAMUNDÍ & Branch_49 & 1 & $8.26 \mathrm{E}-10$ & $1.85 \mathrm{E}-11$ & 0 & $1.24 \mathrm{E}-08$ & $1.86 \mathrm{E}-06$ \\
\hline LA CUMBRE & Branch_50 & 0.70275644 & $1.97 \mathrm{E}-09$ & $1.84 \mathrm{E}-10$ & $4.37 \mathrm{E}-08$ & 0 & $2.72 \mathrm{E}-06$ \\
\hline LA UNIÓN & Branch_51 & 0.474671867 & $5.67 \mathrm{E}-10$ & $5.15 \mathrm{E}-10$ & $1.09 \mathrm{E}-08$ & $1.04 \mathrm{E}-08$ & $1.54 \mathrm{E}-06$ \\
\hline LA VICTORIA & Branch_52 & 0.745108709 & $1.01 \mathrm{E}-09$ & $2.56 \mathrm{E}-10$ & $1.89 \mathrm{E}-08$ & $3.36 \mathrm{E}-08$ & $2.92 \mathrm{E}-06$ \\
\hline PALMIRA & Branch_53 & 0.608003072 & $3.18 \mathrm{E}-10$ & $1.04 \mathrm{E}-10$ & $1.25 \mathrm{E}-09$ & 0 & $1.64 \mathrm{E}-06$ \\
\hline PRADERA & Branch_54 & 0.668104439 & $5.64 \mathrm{E}-10$ & $7.13 \mathrm{E}-10$ & $1.65 \mathrm{E}-08$ & 0 & $1.70 \mathrm{E}-06$ \\
\hline RESTREPO & Branch_55 & 0.822662597 & $1.20 \mathrm{E}-09$ & 0 & $2.99 \mathrm{E}-08$ & $3.48 \mathrm{E}-08$ & $2.40 \mathrm{E}-06$ \\
\hline SAN PEDRO & Branch_56 & 1 & $1.67 \mathrm{E}-09$ & $1.80 \mathrm{E}-10$ & $4.82 \mathrm{E}-08$ & $3.09 \mathrm{E}-08$ & $3.18 \mathrm{E}-06$ \\
\hline TORO & Branch_57 & 0.871172166 & $1.19 \mathrm{E}-09$ & $7.66 \mathrm{E}-11$ & 0 & $8.92 \mathrm{E}-08$ & $3.93 \mathrm{E}-06$ \\
\hline TRUJILLO & Branch_58 & 0.626697857 & $1.43 \mathrm{E}-09$ & $5.80 \mathrm{E}-10$ & $1.92 \mathrm{E}-08$ & $5.27 \mathrm{E}-09$ & $2.60 \mathrm{E}-06$ \\
\hline TULUÁ & Branch_59 & 0.849352055 & $5.60 \mathrm{E}-10$ & 0 & 0 & $9.77 \mathrm{E}-09$ & $1.53 \mathrm{E}-06$ \\
\hline VIJES & Branch_60 & 0.951217159 & $1.46 \mathrm{E}-09$ & $4.03 \mathrm{E}-09$ & 0 & $3.55 \mathrm{E}-08$ & 0 \\
\hline YOTOCO & Branch_61 & 0.704609995 & $1.09 \mathrm{E}-09$ & $9.93 \mathrm{E}-10$ & $2.09 \mathrm{E}-08$ & $2.00 \mathrm{E}-08$ & $2.97 \mathrm{E}-06$ \\
\hline YUMBO & Branch_62 & 0.765692275 & $1.07 \mathrm{E}-09$ & $2.58 \mathrm{E}-10$ & 0 & 0 & $2.22 \mathrm{E}-06$ \\
\hline
\end{tabular}

Anexo 2.

Data-base discriminada por variable para las DMUs (miles de millones de pesos)

\begin{tabular}{|c|c|c|c|c|c|c|}
\hline Municipios & DMU_list & Compras_ByS & Medicamentos & Gto_Admón & Gto_Asistencial & Producción \\
\hline MONTERÍA & Branch_1 & 7265205555 & 2279613612 & 31543605 & 50423265 & 1187457.2 \\
\hline CANALETE & Branch_2 & 565415631.4 & 399387209.2 & 12176407 & 6677438 & 96635.08 \\
\hline CIÉNAGA DE ORO & Branch_3 & 777117028.6 & 1646396364 & 12319436 & 11856192 & 259213.13 \\
\hline MONTELÍBANO & Branch_4 & 1694174503 & 1614767262 & 15486178 & 18344492 & 350938.09 \\
\hline PLANETA RICA & Branch_5 & 825223308.6 & 398885895.1 & 19101890 & 17871282 & 490263.93 \\
\hline PUEBLO NUEVO & Branch_6 & 344686386.8 & 1088664877 & 4125888 & 14223391 & 195849.9 \\
\hline PUERTO ESCONDIDO & Branch_7 & 399225638.3 & 575242955.1 & 4682228 & 20114340 & 99164.32 \\
\hline TIERRALTA & Branch_8 & 3277845984 & 6834089843 & 23182869 & 16089931 & 1377904.24 \\
\hline VALENCIA & Branch_9 & 1177130297 & 1064887371 & 8289480 & 13053416 & 384079.72 \\
\hline SINCELEJO & Branch_10 & 3166760140 & 1043063022 & 39486620 & 45393374 & 856626.11 \\
\hline BUENAVISTA & Branch_11 & 223423468.1 & 112386060.8 & 9502667 & 11659335 & 54991.92 \\
\hline CAIMITO & Branch_12 & 362005703.9 & 45026500.95 & 32060298 & 14957292 & 124065.75 \\
\hline COROZAL & Branch_13 & 932679140.9 & 130970520.7 & 12908269 & 9818966 & 393177.17 \\
\hline EL ROBLE & Branch_14 & 127840307.5 & 84002149.21 & 10454414 & 15644012 & 35257.95 \\
\hline GALERAS & Branch_15 & 275569567.6 & 92444258.87 & 15341988 & 31349920 & 102606.36 \\
\hline MAJAGUAL & Branch_16 & 1510977183 & 358902332.3 & 12985443 & 23592873 & 157781.72 \\
\hline MORROA & Branch_17 & 173736018.1 & 73506806.11 & 8522335 & 11333704 & 74003.05 \\
\hline PALMITO & Branch_18 & 159596293.3 & 285258773.2 & 13224867 & 12783023 & 94041.17 \\
\hline SAMPUÉS & Branch_19 & 672946746.6 & 342062554.1 & 15355590 & 22321184 & 206877.53 \\
\hline SAN BENITO ABAD & Branch_20 & 452495465.2 & 779663338.5 & 10314088 & 11290557 & 284387.66 \\
\hline
\end{tabular}

100

PODIUM No. 39, Junio 2021, pp. 83-102

(C) Universidad Espíritu Santo - UEES

ISSN: 1390-5473 e-ISSN: 2588-0969 
Eficiencia hospitalaria en regiones colombianas con percepción de corrupción, mediante técnica DEA

\begin{tabular}{|c|c|c|c|c|c|c|}
\hline Municipios & DMU_list & Compras_ByS & Medicamentos & Gto_Admón & Gto_Asistencial & Producción \\
\hline $\begin{array}{l}\text { SAN JUAN DE } \\
\text { BETULIA }\end{array}$ & Branch_21 & 408171137.6 & 62882309.05 & 5099655 & 2918839 & 76045.92 \\
\hline SAN MARCOS & Branch_22 & 830914711 & 320973578 & 21958897 & 28056667 & 159453.25 \\
\hline SAN ONOFRE & Branch_23 & 1425853015 & 260670011.9 & 13045244 & 12574838 & 195531.09 \\
\hline SAN PEDRO & Branch_24 & 335451081.7 & 260413209.9 & 9465135 & 13258742 & 215572.01 \\
\hline SAN LUIS DE SINCÉ & Branch_25 & 444206692.5 & 127482361.8 & 20899564 & 26668814 & 165780.38 \\
\hline SUCRE & Branch_26 & 1179261169 & 165366447.6 & 21066912 & 21781582 & 121244.68 \\
\hline SANTIAGO DE TOLÚ & Branch_27 & 417039786.1 & 123540290 & 13429093 & 16627086 & 109711.12 \\
\hline TOLÚ VIEJO & Branch_28 & 188511514.6 & 254492997.4 & 21001579 & 30611906 & 64197.55 \\
\hline CALI & Branch_29 & 1176695701 & 856019207.3 & 204456934 & 88940925 & 728532.49 \\
\hline ALCALÁ & Branch_30 & 175518282 & 261330824 & 12888685 & 15786644 & 158154.29 \\
\hline ANDALUCÍA & Branch_31 & 328420763.3 & 247284213.9 & 21600230 & 25040159 & 144951.27 \\
\hline ANSERMANUEVO & Branch_32 & 468118628.4 & 461931393.4 & 14581798 & 20306785 & 131239.74 \\
\hline ARGELIA & Branch_33 & 24905377.79 & 162162588.3 & 12151931 & 7372263 & 30648.35 \\
\hline BOLÍVAR & Branch_34 & 327882031.3 & 430430908.7 & 14436958 & 7555618 & 75699.51 \\
\hline BUENAVENTURA & Branch_35 & 1077868048 & 635333238.8 & 13969874 & 16237274 & 36977.69 \\
\hline $\begin{array}{c}\text { GUADALAJARA DE } \\
\text { BUGA }\end{array}$ & Branch_36 & 987983284.7 & 818088538.2 & 30917519 & 18054250 & 344869.17 \\
\hline CAICEDONIA & Branch_37 & 695740437.9 & 680122726.5 & 22442629 & 21249509 & 250334.11 \\
\hline CALIMA & Branch_38 & 330099040.9 & 362562623.5 & 19099059 & 28848081 & 88879.2 \\
\hline CANDELARIA & Branch_39 & 1073164066 & 446004369.2 & 19139000 & 27616000 & 276047.4 \\
\hline CARTAGO & Branch_40 & 757732577.4 & 2019735981 & 25331233 & 21117212 & 396651.63 \\
\hline DAGUA & Branch_41 & 669221962.4 & 658389013.4 & 90222391 & 22417829 & 172274.19 \\
\hline EL ÁGUILA & Branch_42 & 282302413.3 & 266441169.4 & 12016442 & 14462469 & 50254.38 \\
\hline EL CAIRO & Branch_43 & 253311947.3 & 118984951.8 & 13589540 & 12139856 & 44504.8 \\
\hline EL CERRITO & Branch_44 & 495375708.1 & 141000261.4 & 19745659 & 17608060 & 318467.12 \\
\hline EL DOVIO & Branch_45 & 302422126.9 & 273572489.4 & 18769649 & 21853404 & 93073.13 \\
\hline FLORIDA & Branch_46 & 773884853.8 & 911525050.4 & 13377000 & 19470000 & 254318.57 \\
\hline GINEBRA & Branch_47 & 303943598.4 & 376262212.6 & 17077813 & 19280195 & 126601.04 \\
\hline GUACARÍ & Branch_48 & 344013508.5 & 241057520.2 & 19143964 & 16582550 & 159792.28 \\
\hline JAMUNDÍ & Branch_49 & 578718931.7 & 1691084092 & 36756961 & 39483481 & 538060.34 \\
\hline LA CUMBRE & Branch_50 & 170942474.4 & 270321398.2 & 14067615 & 16800584 & 65023.91 \\
\hline LA UNIÓN & Branch_51 & 821555962 & 303873108.8 & 17869000 & 17679000 & 157152.7 \\
\hline LA VICTORIA & Branch_52 & 340886439.4 & 238006341.9 & 14034250 & 9804297 & 118084.27 \\
\hline PALMIRA & Branch_53 & 2073788496 & 2737875835 & 43702880 & 40801288 & 660864.23 \\
\hline PRADERA & Branch_54 & 777693830.8 & 260110476.9 & 22796100 & 39549879 & 296403.27 \\
\hline RESTREPO & Branch_55 & 304419770.9 & 302960442.6 & 12072770 & 7819430 & 108037.77 \\
\hline SAN PEDRO & Branch_56 & 91620152.48 & 148037794.6 & 12746996 & 6689684 & 67219.8 \\
\hline TORO & Branch_57 & 255134419.8 & 297245734.4 & 16078083 & 7539713 & 106279.5 \\
\hline TRUJILLO & Branch_58 & 272189994.5 & 296554973.7 & 17917695 & 17890330 & 117070.98 \\
\hline TULUÁ & Branch_59 & 1202729285 & 3310146417 & 44309657 & 33359400 & 629106.91 \\
\hline VIJES & Branch_60 & 273183346.1 & 78827706.17 & 7960631 & 8022962 & 51156.46 \\
\hline Yотосо & Branch_61 & 278577399.3 & 180213504.8 & 14696796 & 10481903 & 86538.96 \\
\hline YUMBO & Branch_62 & 703383954.7 & 963691589.6 & 48970530 & 52638544 & 408360.56 \\
\hline
\end{tabular}

\section{1}

PODIUM No. 39, Junio 2021, pp. 83-102

(c) Universidad Espíritu Santo - UEES

ISSN: 1390-5473 e-ISSN: 2588-0969 
